\title{
Linear non-equilibrium thermodynamics of human voluntary behavior: a canonical-dissipative Fokker-Planck equation approach involving potentials beyond the harmonic oscillator case
}

\author{
J.M. Gordon ${ }^{11}$, S. Kim ${ }^{11}$, T.D. Frank ${ }^{112}$ \\ ${ }^{1}$ CESPA, Department of Psychology, University of Connecticut, 406 Babbidge Road, Storrs, CT 06269, USA \\ 2 Department of Physics, University of Connecticut, 2152 Hillside Road, Storrs, CT 06269, USA
}

Received June 7, 2016

\begin{abstract}
A novel experimental paradigm and a novel modelling approach are presented to investigate oscillatory human motor performance by means of a key concept from condensed matter physics, namely, thermodynamic state variables. To this end, in the novel experimental paradigm participants performed pendulum swinging movements at self-selected oscillation frequencies in contrast to earlier studies in which pacing signals were used. Moreover, in the novel modelling approach, a canonical-dissipative limit cycle oscillator model was used with a conservative part that accounts for nonharmonic oscillator components in contrast to earlier studies in which only harmonic components were considered. Consistent with the Landau theory of magnetic phase transitions, we found that the oscillator model free energy decayed when participants performed oscillations further and further away from the Hopf bifurcation point of the canonical-dissipative limit cycle oscillator.
\end{abstract}

Key words: physics of life, human behavior, canonical-dissipative systems, thermodynamic state variables

PACS: 05.40.Jc, 05.45.-a, 05.70.Ce, 05.70.Ln, 64.70.qd, 87.19.rs

In view of the success of physics in general and condensed matter physics in particular, the question has been debated whether concepts from physics and condensed matter physics can be generalized to describe not only equilibrium systems but also non-equilibrium systems (e.g. [1 2]). The canonicaldissipative (CD) approach to non-equilibrium systems [3]-6] in this context plays an important role because it exhibits key features of condensed matter physics such as Hamiltonian mechanics and thermodynamic state variables [7]. Moreover, in a series of recent experimental studies it has been shown that the $\mathrm{CD}$ approach can be applied to characterize the performance of humans - as paradigmatic nonequilibrium systems - in simple oscillatory motor control tasks [8]-10]. However, these earlier studies were subjected to two severe limitations. First, the experimental studies involved a pacing signal which is inconsistent with the model of an autonomous limit-cycle oscillator. Although in the study by Kim et al. [10], the impact of the pacing signal was greatly reduced, the question arises whether or not the experimental design can be improved to do without any pacing signal. Second, all three studies used the standard CD oscillator model exhibiting the conservative part of a harmonic oscillator. In this regard, the question arises how to go beyond the harmonic oscillator case. In what follows we will report an experimental design in which the pacing signal was completely absent. Moreover, we will consider conservative parts of the CD modelling approach that correspond to nonharmonic oscillators.

In the experiment participants were sitting in a chair with their right arm fixed to a custom armrest, allowing them to swing a pendulum ( $40 \mathrm{~cm}$ rod, cylindrical weight positioned $2.5 \mathrm{~cm}$ from end, $303 \mathrm{~g}$ total weight) with resonant frequency of $1 \mathrm{~Hz}(6.28 \mathrm{rad} / \mathrm{s})$ with their right hand about the wrist. Goniometers were attached to the arm to capture the wrist angle (measured in radiants) while swinging the pendulum (Biometrics, $100 \mathrm{~Hz}$ sampling rate). For the duration of each one-minute trial, participants were instructed 
to swing at one of three paces: comfortable, fast, or slow (3 conditions, 3 repetitions each, presentation randomized). Rather than synchronize their swinging to an external signal (i.e., a metronome) for reference, participants determined their own pacing for all trials, scaled relative to their natural ("comfortable") swinging frequency. Practice trials ensured that participants could generate 3 different swinging paces and sustain them for one minute each. Practice began with the "comfortable" condition. A tablet (Android, Nexus 10) was used to approximate the participant pacing in beats per minute (BPMs), where a "beat" was marked at the furthest point of the pendulum's arc. Average BPM was also tracked for fast and slow conditions. "Fast" swinging was required to be at least $20 \%$ faster than their comfortable pace, and "slow" was likewise required to be at least $20 \%$ slower in speed. If pacing during a slow or fast trial failed to exceed this $20 \%$ difference, participants were notified and prompted to increase or decrease their pace accordingly. After practice, most participants completed the experiment successfully and without further corrections from the experimenter. The experimental design allowed us to investigate and manipulate voluntary, oscillatory human motor performance without the use of any external pacing signal. To this end, the oscillatory goniometer signal was evaluated.

Let $x$ denote goniometer (wrist angle) signal in radiants and let $v$ denote the corresponding velocity $v=\mathrm{d} x / \mathrm{d} t$. Let $\mathbf{r}=(x, v)$ denote the state vector of the oscillatory system and $H(x, v)$ a Hamiltonian function that will be specified later. The Langevin equation [11] of the CD model for the self-oscillator reads [3, 7, 12]

$$
\frac{\mathrm{d}}{\mathrm{d} t} \mathbf{r}=\mathbf{I}-\gamma \nabla_{\nu} \Phi+\left(\begin{array}{c}
0 \\
\sqrt{D} \Gamma(t)
\end{array}\right)
$$

where $\mathbf{I}=(\partial H / \partial v,-\partial H / \partial x)$ (from a dynamical system's perspective) is the conservative "force" acting on the oscillator. In equation (1) $\nabla_{\nu}=(0, \partial / \partial v)$ is the incomplete Nabla operator and $\Phi=\Phi(H)$ is a function of $H$. The parameter $D \geqslant 0$ denotes the diffusion coefficient and is composed of the damping parameter $\gamma \geqslant 0$ and the noise amplitude $\theta \geqslant 0$ like $D=\gamma \theta$. Finally, $\Gamma(t)$ denotes a Langevin force [11] normalized like $\left\langle\Gamma(t) \Gamma\left(t^{\prime}\right)\right\rangle=2 \delta\left(t-t^{\prime}\right)$. Here and in what follows $\delta(z)$ represents the Dirac delta function and $\langle\cdot\rangle$ stands for ensemble averaging. In the conservative case (i.e., for $\gamma=0 \Rightarrow D=0$ ) equation (1) corresponds to a Hamiltonian dynamics. Therefore, we interpret $H$ as Hamiltonian function of the oscillator. The probability density of the state variables $x$ and $v$ is defined by $P(x, v, t)=\langle\delta(x-x(t)) \delta(v-v(t))\rangle$. From equation (1) it follows that $P(x, v, t)$ satisfies the so-called free energy Fokker-Planck equation [7] of the form

$$
\frac{\partial}{\partial t} P=-\nabla \cdot \mathbf{I} P+\nabla \cdot M \cdot P \nabla \frac{\delta F_{\mathrm{NE}}}{\delta P}
$$

In equation (2) we have the complete Nabla operator $\nabla=(\partial / \partial x, \partial / \partial v)$ and $\delta F_{\mathrm{NE}} / \delta P$ denotes the variational derivative of the functional $F_{\mathrm{NE}}$ with respect to $P$. The functional $F_{\mathrm{NE}}$ itself is one of the three (non-equilibrium) thermodynamic variables of the CD model and denotes the non-equilibrium free energy. The two other variables are the non-equilibrium internal energy $U_{\mathrm{NL}}$ and the statistical entropy $S$ defined by [7]

$$
S=-\int P \ln P \mathrm{~d} x \mathrm{~d} v, \quad U_{\mathrm{NL}}=\langle\Phi\rangle, \quad F_{\mathrm{NE}}=U_{\mathrm{NL}}-\theta S .
$$

Moreover, $M$ denotes a $2 \times 2$ mobility matrix with coefficients $M_{22}=\gamma$ and $M_{j k}=0$ otherwise. From equation (3) it follows that $\theta$ plays the role of a non-equilibrium temperature. The stationary solution of equation (2) in phase space $(x, v)$ reads

$$
P(x, v)=\frac{1}{Z_{x v}} \exp \left[-\frac{\Phi(H(x, v))}{\theta}\right],
$$

where $Z_{x v}$ is a normalization constant. Let us introduce the probability density of the Hamiltonian function $H$ like $P(H)=\int \delta(H-H(x, v)) P(x, v) \mathrm{d} x \mathrm{~d} v$. We obtain

$$
P(H)=\frac{1}{Z_{H}} \exp \left[-\frac{\Phi(H)}{\theta}\right],
$$

where $Z_{H}$ is a normalization constant again. Note that the normalization constants $Z_{x v}$ and $Z_{H}$ are related to each other [6]. Importantly, $P(H)$ is maximal at the energy value $H$ for which $\Phi$ is minimal. 
Therefore, $\Phi(H)$ can be regarded as non-equilibrium potential of $H$. In addition, if we put $\Phi=(H-B)^{2} / 2$, where $B$ is a parameter that will be discussed below, then with respect to $P(H)$ the variables $F_{\mathrm{NL}}, U_{\mathrm{NL}}$ and $S$ defined by equation (3) can be calculated from $B$ and $\theta$ like [9, 10]

$$
U_{\mathrm{NE}}=\frac{\theta}{2}\left\{1-\frac{B \exp \left[-B^{2} /(2 \theta)\right]}{Z_{H}}\right\}, \quad S=\ln Z_{H}+\frac{U_{H}^{\mathrm{NE}}}{\theta}, \quad F_{\mathrm{NE}}=-\theta \ln Z_{H}
$$

with $Z_{H}=\sqrt{2 \pi \theta} w(B / \sqrt{\theta})$, where $w(s)$ is the error function $w(s)=\int_{-\infty}^{s} \exp \left(-z^{2} / 2\right) \mathrm{d} z$.

Let us dwell on the interpretation of $\Phi$. Let us put $\gamma>0$ but $\theta=0 \Rightarrow D=0$ such that

$$
\frac{\mathrm{d}}{\mathrm{d} t} x=\frac{\partial H}{\partial v}, \quad \frac{\mathrm{d}}{\mathrm{d} t} v=-\frac{\partial H}{\partial x}-\gamma \frac{\partial \Phi}{\partial v} \quad \Rightarrow \quad \frac{\mathrm{d}}{\mathrm{d} t} \Phi=-\gamma\left(\frac{\partial \Phi}{\partial v}\right)^{2} \leqslant 0 .
$$

That is, $\Phi$ is a nonincreasing function of time. Frequently, the special case of a quadratic function

$$
\Phi(H)=\frac{(H-B)^{2}}{2}
$$

has been discussed in the literature (e.g. [3]). In this case, equation (7) reads

$$
\frac{\mathrm{d}}{\mathrm{d} t} x=\frac{\partial H}{\partial v}, \quad \frac{\mathrm{d}}{\mathrm{d} t} v=-\frac{\partial H}{\partial x}-\gamma \frac{\partial H}{\partial v}(H-B) \quad \Rightarrow \quad \frac{\mathrm{d}}{\mathrm{d} t} H=-\gamma\left(\frac{\partial H}{\partial v}\right)^{2}(H-B) .
$$

Let us assume $H$ is bounded from below. For the sake of simplicity we assume that (i) $H \geqslant 0$ holds for all $x, v$, (ii) $H$ is a smooth function defined on the phase space $x, v$, and there exists at least one pair $x$ (min), $v$ (min) such that $H=0$. Furthermore, let us assume that if $H>0$ holds then trajectories $x(t), v(t)$ of equation (9) are such that $\partial H / \partial v=0$ holds only for a discrete set $K$ of time points $t_{1}<t_{2}<t_{3} \ldots$. Consequently, provided that $H>0$ and $H \neq B$ holds, then we have $\mathrm{d} H / \mathrm{d} t<0$ if $t$ is not in the set $K$. This implies that $H$ and $\Phi$ exhibit asymptotically stable fixed points at $H=0$ and $\Phi=B^{2} / 2$ for $B \leqslant 0$ and $H=B$ and $\Phi=$ 0 for $B>0$. In particular, for both cases, the potential $\Phi$ converges to its minimum value $\Phi_{\min }=\min _{H}(\Phi)$.

So far, in applications of the model (1) to experimental research on human motor behavior, the focus has been on the harmonic oscillator case [8-10]

$$
H=\frac{v^{2}}{2}+\frac{\omega^{2} x^{2}}{2}
$$

Taking $B$ as a control parameter of the deterministic oscillatory system (1) with $\theta=0$, then there is a Hopf bifurcation point at $B=0$. In the experiment, the parameter $B$ reflects the intention of a participant to swing $(B>0)$ or not to swing $(B \leqslant 0)$ the pendulum. As far as the CD oscillator model is concerned, for $B \leqslant 0$ equation (9) exhibits an asymptotically stable fixed point at $(x, v)=(0,0)$ with $H=0$. By contrast, for $B>0$ the fixed point $(x, v)=(0,0)$ is an unstable focus. There exists a stable limit cycle characterized by the harmonic oscillator equation $\mathrm{d}^{2} x / \mathrm{d} t^{2}=-\omega^{2} x$ and an oscillation amplitude $A$ given by $\omega^{2} A^{2} / 2=$ $B$ [8]. Consequently, on the limit cycle we have $x(t)=\omega^{-1} \sqrt{2 B} \cos (\omega t+\varphi)$, where $\varphi$ is an arbitrary phase.

Let us generalize the considerations in order to consider nonharmonic limit cycles. To this end, we consider Hamiltonian functions of the form

$$
H=\frac{v^{2}}{2}+V(x), \quad V(x)=V_{0}(x)+V_{1}(x) .
$$

$V_{0}$ is considered as baseline oscillator potential and $V_{1}$ is considered as a correction term. However, we do not require that $V_{1}$ should denote a small correction term. By contrast, we require that the baseline potential should be quadratic like

$$
V_{0}(x)=k_{1} x+k_{2} \frac{x^{2}}{2}
$$

with $k_{2}>0$, whereas $V_{1}$ accounts for contributions different from the linear and quadratic cases. In order to make sure that $V(x)$ is globally stable such that non-equilibrium thermodynamic variables $F_{\mathrm{NE}}$ 
and $U_{\mathrm{NL}}$ and the statistical entropy $S$ exist we require that $V_{1}(|x| \rightarrow \infty)=0$ should hold. In what follows, we will use

$$
V_{1}(x)=\exp \left(-x^{2} / 2\right) \sum_{j=2}^{N} k_{j+1} x^{j+1} .
$$

In fact, for our purposes any other function $V_{1}$ could be used provided that $V_{1}(|x| \rightarrow \infty)=0$ holds and $V_{1}$ is linearly independent with respect to the linear and quadratic functions $x$ and $x^{2}$, respectively. Let us consider the conservative case $\gamma=0 \Rightarrow D=0$ again. From $\mathrm{d} \mathbf{r} / \mathrm{d} t=\mathbf{I}$ and equations (11,13) it then follows that

$$
\frac{\mathrm{d}^{2}}{\mathrm{~d} t^{2}} x=-k_{1}-k_{2} x-\exp \left(-x^{2} / 2\right) \sum_{j=2}^{N} k_{n+1}\left[(n+1) x^{n}-x^{n+2}\right] \text {. }
$$

Therefore, in the dissipative, deterministic case $\gamma>0$ with $\theta=0$ for appropriately chosen parameters $k_{j}$ with $N>2$, the model (9) can exhibit stable limit cycles that describe nonharmonic oscillatory behavior. Importantly, in the fully dissipative case $\gamma>0$ with $\theta>0$, the CD oscillator model (1) can be used to determine the non-equilibrium thermodynamic variables $F_{\mathrm{NE}}$ and $U_{\mathrm{NL}}$ and the statistical entropy $S$ of the self-oscillator.

The variables $F_{\mathrm{NE}}, U_{\mathrm{NL}}$, and $S$ were estimated from experimental data in a three-step approach. First, the model parameters of the Hamiltonian $k_{1}, \ldots, k_{n}$ were determined using linear regression analysis [13. [14]. Second, the canonical-dissipative parameters $\theta$ and $B$ were estimated. To this end, we followed [8, 10] and calculated the Hamiltonian energy values $H$ from equations 11,13 and the experimentally observed time series $x(t)$ and $v(t)=\mathrm{d} x / \mathrm{d} t$ using the parameters estimates $k_{1}, \ldots, k_{n}$. Third, following again the earlier work [9, 10], we calculated $F_{\mathrm{NE}}, U_{\mathrm{NL}}$, and $S$ from equation (6) and the estimated values of $\theta$ and $B$.

A total of ten participants (undergraduate students from the University of Connecticut who received partial course credit for their participation) were tested. Experimental procedures (as described above) were approved by the University's Institutional Review Board (IRB). Two participants performed oscillatory movements that were subjected to a great amount of jerk and could not be classified with the aforementioned data analysis procedure as limit cycle oscillations. The data of those two participants were discarded. Only the data from the remaining eight participants were evaluated. Two models were tested: the baseline model involving only $V_{0}$ and the parameters $k_{1}, k_{2}$ (for the harmonic oscillator case) and the $N=5$ model (for the nonharmonic oscillator case) involving parameters $k_{1}, \ldots, k_{6}$ and terms up to the order $x^{6}$.

Table 1 shows descriptive measures (oscillation frequency and amplitude) of the observed oscillatory movements for the three frequency conditions. As expected, oscillation frequency $\omega$ increased significantly across the frequency conditions [ $p<0.01, \mathrm{~F}(2.14)=43.683$ ]. The amplitude $A$ showed a U-shaped pattern with the minimum at the comfortable condition. The effect of the frequency on $A$ was statistically significant [ $p<0.05, \mathrm{~F}(2.14)=5.045]$. Table 2 shows the improvement $\Delta R^{2}$ of the $R^{2}$ goodness of fit scores when comparing the nonharmonic case with the harmonic case. By definition of the fitting procedure,

Table 1. Angular frequencies $\omega$ and oscillation amplitudes $A$ as functions of the three frequency (speed) conditions (slow, comfortable, fast). Angular frequencies were determined as peak frequencies observed in the Fourier spectrum of the movement trajectories $x(t)$. Amplitudes were calculated from the difference between averaged maximal and averaged minimal values of $x(t): A=[\overline{\text { Peaks(MAX) }}-$ $\overline{\text { Peaks(MIN) }}] / 2$. Standard errors in parenthesis.

\begin{tabular}{|c|c|c|c|}
\hline \hline & Slow & Comfortable & Fast \\
\hline \hline$\omega[\mathrm{rad} / \mathrm{s}]$ & $5.35(0.36)$ & $7.01(0.47)$ & $9.10(0.71)$ \\
\hline$A$ [a.u.] & $0.27(0.03)$ & $0.25(0.03)$ & $0.28(0.03)$ \\
\hline \hline
\end{tabular}

Table 2. Improvements of model fits quantified by $R^{2}$-improvements scores $\Delta R^{2}$ observed for the three experimental frequency (speed) conditions.

\begin{tabular}{|c|c|c|c|}
\hline \hline & Slow & Comfortable & Fast \\
\hline \hline$\Delta R^{2} \% 0$ & 1.6 & 2.6 & 1.4 \\
\hline \hline
\end{tabular}


the $R^{2}$ scores of the more comprehensive model (nonharmonic case) should exceed the $R^{2}$ scores of the less comprehensive model (harmonic case) such that $\Delta R^{2}>0$. The $\Delta R^{2}$ scores reported in table 2 reflect this fundamental feature of regression models. For our purposes, table 2 demonstrates that we were able to improve the fits of the self-oscillator models by taking nonharmonic contributions into account.

Table 3 presents the bifurcation parameter $B$ as a function of frequency and model type. Consistent with the previous studies [8]-10], there was a statistically significant increase of $B$ from slow to fast oscillations speeds [harmonic case: $p<0.01, \mathrm{~F}(2.14)=11.722$; nonharmonic case: $p<0.01, \mathrm{~F}(2.14)=10.694]$. That is, $B$ increased with increasing oscillation frequency. Importantly, the same pattern was qualitatively observed for both models. Table 4 displays the statistical entropy $S$ and the non-equilibrium thermodynamical variables $U_{\mathrm{NL}}$ and $F_{\mathrm{NL}}$ for the models and frequency conditions. Entropy $S$ and internal energy $U_{\mathrm{NL}}$ scores increased significantly as functions of oscillation frequency [harmonic case: $p<0.01$, $\mathrm{F}(2.14)=35.804$ for $S$ and $p<0.05, \mathrm{~F}(2.14)=3.991$ for $U_{\mathrm{NL}}$; nonharmonic case: $p<0.01, \mathrm{~F}(2.14)=36.582$ for $S$ and $p=0.06, \mathrm{~F}(2.14)=3.503$ for $U_{\mathrm{NL}}$. Note that the increase of $U_{\mathrm{NL}}$ in the nonharmonic case was only marginally statistically significant. Consistent with the previous studies [9, 10, 15], the non-equilibrium free energy $F_{\mathrm{NL}}$ decreased when oscillation frequency increased. The effect was marginally statistically significant [harmonic case: $p=0.07, \mathrm{~F}(2.14)=3.188$; nonharmonic case: $p=0.09, \mathrm{~F}(2.14)=2.810$ ]. We will return to this issue below.

Table 3. Bifurcation parameter $B$ obtained for different frequency (speed) conditions as obtained from the harmonic and nonharmonic model-based data analysis. Standard errors in parenthesis.

\begin{tabular}{|l||c|c|c|}
\hline \hline Case & $\begin{array}{c}B \text { (Slow) } \\
\text { [a.u.] }\end{array}$ & $\begin{array}{c}B \text { (Comfortable) } \\
\text { [a.u.] }\end{array}$ & $\begin{array}{c}B \text { (Fast) } \\
\text { [a.u.] }\end{array}$ \\
\hline \hline Harmonic & $1.3(0.3)$ & $2.1(0.6)$ & $4.1(1.1)$ \\
\hline Nonharmonic & $1.3(0.3)$ & $2.2(0.7)$ & $4.5(1.3)$ \\
\hline \hline
\end{tabular}

Table 4. Variables $S, U_{\mathrm{NL}}$, and $F_{\mathrm{NL}}$ as functions of the frequency (speed) conditions (S=slow, C=comfortable, $\mathrm{F}=$ fast) and model type. Standard errors in parenthesis.

\begin{tabular}{|l||c|c|c|c|c|c|c|c|c|}
\hline \hline $\begin{array}{l}\text { Model- } \\
\text { type }\end{array}$ & $\begin{array}{c}S(\mathrm{~S}) \\
\text { [a.u.] }\end{array}$ & $\begin{array}{c}S(\mathrm{C}) \\
\text { [a.u.] }\end{array}$ & $\begin{array}{c}S(\mathrm{~F}) \\
\text { [a.u.] }\end{array}$ & $\begin{array}{c}U_{\mathrm{NL}}(\mathrm{S}) \\
\text { [a.u.] }\end{array}$ & $\begin{array}{c}U_{\mathrm{NL}}(\mathrm{C}) \\
\text { [a.u.] }\end{array}$ & $\begin{array}{c}U_{\mathrm{NL}}(\mathrm{F}) \\
\text { [a.u.] }\end{array}$ & $\begin{array}{c}F_{\mathrm{NL}}(\mathrm{S}) \\
\text { [a.u.] }\end{array}$ & $\begin{array}{c}F_{\mathrm{NL}}(\mathrm{C}) \\
\text { [a.u.] }\end{array}$ & $\begin{array}{c}F_{\mathrm{NL}}(\mathrm{F}) \\
\text { [a.u.] }\end{array}$ \\
\hline \hline Harmonic & 1.1 & 1.3 & 1.9 & 0.5 & 0.9 & 2.9 & -1.1 & -3.2 & -14.6 \\
& $(0.2)$ & $(0.2)$ & $(0.3)$ & $(0.1)$ & $(0.5)$ & $(1.4)$ & $(0.6)$ & $(2.1)$ & $(8.2)$ \\
\hline Non- & 1.1 & 1.3 & 1.8 & 0.4 & 0.8 & 2.8 & -1.0 & -2.8 & -13.9 \\
harmonic & $(0.2)$ & $(0.2)$ & $(0.3)$ & $(0.1)$ & $(0.4)$ & $(1.4)$ & $(0.5)$ & $(1.9)$ & $(8.1)$ \\
\hline \hline
\end{tabular}

Our experimental design allowed us to examine and manipulate voluntary human performance in an oscillatory motor control task without the use of an external pacing signal. Moreover, we were able to account for nonharmonic oscillator contributions in the conservative part of the CD modelling approach. As expected, the nonharmonic models matched the data better than the harmonic models. Importantly, the thermodynamic state variables showed for the harmonic and nonharmonic analyses the same kind of patterns across the three frequency conditions. Therefore, it seems that (at least for the experimental paradigm of the present study) the thermodynamic variables are not affected by the model type.

The pumping parameter $B$ increased with oscillation frequency. It has been suggested that $B$ measures the distance to the Hopf bifurcation point of the canonical-dissipative self-oscillator [9, 10]. Accordingly, the non-equilibrium free energy decayed when the tested "human" self-oscillators operated further away from their bifurcation points. It has been argued that this feature is consistent with the decay of the Landau free energy of magnetic phase transitions [16] when the temperature is decreased further below the critical transition temperature [9]10].

For the sample of eight participants, the decay of the non-equilibrium free energy was only marginally statistically significant. However, a detailed inspection of the individual participant data revealed that two of the eight participants showed free energy scores $F_{\mathrm{NL}}$ that were by a factor of 10 larger than the scores obtained for the remaining six participants. Analyzing the non-equilibrium free energy only for 
the remaining "homogeneous" group of six participants, we found for $F_{\mathrm{NL}}$ the same qualitative pattern as for the whole group: the free energy decayed with an increasing oscillation frequency. However, for this homogeneous subset of participants, the effect of speed was also statistically significant ( $p<0.05$ for both models). Future experimental work may be conducted to explore this participant effect in more detail.

\title{
Acknowledgements
}

Preparation of this manuscript was supported in part by National Science Foundation under the INSPIRE track, grant BCS-SBE-1344275.

\section{References}

1. Glansdorff P., Prigogine I., Thermodynamic Theory of Structure, Stability, and Fluctuations, John Wiley and Sons, New York, 1971.

2. Haken H., Synergetics. An Introduction, Springer, Berlin, 1977.

3. Ebeling W., Sokolov I.M., Statistical Thermodynamics and Stochastic Theory of Nonequilibrium Systems, World Scientific, Singapore, 2004.

4. Haken H., Z. Phys., 1973, 263, 267; doi 10.1007/BF01391586

5. Graham R., In: Quantum Statistics in Optics and Solid-State Physics. Springer Tracts in Modern Physics Vol. 66, Höhler G. (Ed.), Springer, Berlin, 1973, 1-97.

6. Mongkolsakulvong S., Frank T.D., Condens. Matter Phys., 2010, 13, 13001; doi 10.5488/CMP.13.13001

7. Frank T.D., Nonlinear Fokker-Planck Equations: Fundamentals and Applications, Springer, Berlin, 2005.

8. Dotov D.G., Frank T.D., Motor Control, 2011, 15, 550.

9. Dotov D.G., Kim S., Frank T.D., Biosystems, 2015, 128, 26; doi 10.1016/j.biosystems.2015.01.002

10. Kim S., Gordon J.M., Frank T.D., Open Syst. Inf. Dyn., 2015, 22, 1550007; doi 10.1142/S1230161215500079

11. Risken H., The Fokker-Planck Equation: Methods of Solution and Applications, Springer, Berlin, 1989.

12. Romanczuk P., Bär M., Ebeling W., Lindner B., Schimansky-Geier L., Eur. Phys. J. Spec. Top., 2012, 202, 1; doi $10.1140 /$ epjst/e2012-01529-y

13. Frank T.D., Int. J. Mod. Phys. B, 2011, 25, 243; doi 10.1142/S0217979211057712.

14. Silva P., Moreno M., Fonseca S., Turvey M.T., Neurosci. Lett., 2009, 429, 64; doi 10.1016/j.neulet.2007.09.066.

15. Frank T.D., Friedrich R., Beek P.J., Phys. Rev. E, 2006, 74, 051905; doi 10.1103/PhysRevE.74.051905.

16. Strobl G., Condensed Matter Physics: Crystals, Liquids, Liquid Crystals, and Polymers, Springer, Berlin, 2004.

\section{Лінійна нерівноважна термодинаміка людської поведінки: метод канонічно-дисипативного рівняння Фоккера-Планка 3 потенціалами, що включають ангармонізми}

\author{
Дж.М. Гордон ${ }^{11}$, С.Кім ${ }^{1}$, Т.Д. Франк ${ }^{112}$ \\ 1 CESPA, факультет психології, Університет Коннектикуту, Сторрс, СТ 06269, США \\ 2 Факультет фізики, Університет Коннектикуту, Сторрс, Ст 06269, США
}

\begin{abstract}
Представлено нову експериментальну парадигму та новий підхід до моделювання роботи осциляторного людського двигуна з допомогою ключового концепту фізики конденсованої речовини, а саме - змінних термодинамічного стану. Для цього в новій експериментальній парадигмі учасники здійснювали маятникові коливні рухи з особисто вибраними частотами, що відмінне від попередніх досліджень, де використовувалися синхронізуючі сигнали. У новому підході моделювання використано канонічно-дисипативну границю циклічно осциляційної моделі з консервативною частиною, що враховує негармонічні коливні компоненти - у цьому інша відмінність від попередніх досліджень, у яких розглядалися лише гармонічні компоненти. В узгодженні з теорією Ландау магнітних фазових переходів ми знайшли, що вільна енергія осциляційної моделі зменшується, коли учасники підтримують коливання з віддаленням від точки біфуркації Хопфа для канонічно-дисипативної границі циклічного осцилятора.
\end{abstract}

Ключові слова: фізика життя, людська поведінка, канонічно-дисипативні системи, змінні термодинамічного стану 